\section{Mycobacterium mucogenicum Peritonitis in a Continuous Ambulatory Peritoneal Dialysis Patient}

\section{Editor:}

Mycobacterium mucogenicum (formerly Mycobacterium chelonae-like organism) is a recently characterized, rapidly growing, atypical nontuberculous mycobacterium (NTM) that occasionally causes human infection (1). We present a patient on continuous ambulatory peritoneal dialysis (CAPD) who experienced peritonitis because of an atypical mycobacterium identified as M. mucogenicum.

A 52-year-old diabetic man on CAPD (2.5-L exchanges 3 - 4 times daily) for 3.5 years presented with nausea, vomiting, abdominal pain, and altered sensorium for 2 days, together with cloudy effluent. He had a history of left ventricular failure, with intermittent atrial fibrillation, and of 6 previous episodes of peritonitis (recurrent gram-negative Escherichia coli peritonitis, and 1 episode of Staphylococcus aureus peritonitis), which were successfully treated. He weighed $98 \mathrm{~kg}$.

The patient's blood urea was $128 \mathrm{mg} / \mathrm{dL}$; plasma creatinine, $10.1 \mathrm{mg} / \mathrm{dL}$; vitamin $\mathrm{D}, 4.61 \mathrm{ng} / \mathrm{mL}$; highsensitivity C-reactive protein, $42.30 \mathrm{mg} / \mathrm{L}$; total protein, $6.7 \mathrm{~g} / \mathrm{dL}$; serum albumin, $3.1 \mathrm{~g} / \mathrm{dL}$; total white blood cell count, $10300 / \mathrm{mm}^{3}$, with $79.6 \%$ neutrophils, $3.8 \%$ monocytes, $16 \%$ lymphocytes, $0.5 \%$ eosinophils, and $0 \%$ basophils. Effluent cell count was $6300 / \mathrm{mm}^{3}$, with $98 \%$ neutrophils and $2 \%$ lymphocytes. Fluid culture and gram stain were unremarkable.

The patient was initiated on intraperitoneal antibiotics per the protocol recommended by the International Society for Peritoneal Dialysis. Fluid culture showed no fungal elements after 1 month of incubation. Culture on Lowenstein-Jensen medium produced colonies within 3 weeks. Effluent sent for nuclear amplification technique showed the presence of a mycobacterium that was later identified as M. mucogenicum.

The previous antimicrobials were discontinued, and the patient was initiated on rifampin $600 \mathrm{mg}$, pyrazinamide $1 \mathrm{~g}$, isoniazid $300 \mathrm{mg}$, ciprofloxacin $500 \mathrm{mg}$ twice daily with vitamin $B_{6}$. Vitamin $D$ levels in this patient were very low, which we suspect may have in the past predisposed him to repeated episodes of peritonitis because of defective immunity. Thus, we also instituted vitamin D therapy. A few days after the start of the new therapy, the effluent cell count was $4200 / \mathrm{mm}^{3}$, with $97 \%$ polymorphonuclear cells and $3 \%$ lymphocytes. After 7 days, the effluent became clear. The patient continued his medication for 2.5 months, and he continues on peritoneal dialysis 6 months later with no ultrafiltration failure.
Many laboratory characteristics of $M$. mucogenicum are similar to those of $M$. chelonae (formerly M. chelonae chelonae), including failure to grow on medium containing $5 \%$ sodium chloride, inability to reduce nitrate, borderline or negative reaction in the iron uptake test, and utilization of citrate as a sole carbon source. The organism was therefore tentatively designated $M$. chelonae-like organism by Silcox et al., after which it was finally named M. mucogenicum (1).

M. mucogenicum is a weakly gram-positive, acid-fast, beady bacillus that can be detected by acid-fast staining using Ziehl-Neelsen stain or, more recently, auramine rhodamine stain with fluorescent microscopy. In culture, it is characterized by growth occurring as early as 7 days after incubation in Lowenstein-Jensen medium. It produces mucoid colonies that are non-pigment-producing and urease-reducing. Hence, they are classified into the group of rapid growers.

M. mucogenicum is frequently isolated from tap water and respiratory specimens, with little clinical significance apart from posttraumatic skin infections and catheterrelated sepsis. The organism has been associated mainly with nosocomial infections, including, in 1976, peritonitis from contamination of automated peritoneal dialysis machines (1), bacterial infection traced to tap water used in hemodialysis, bacteremia from the Hickman line in an immunocompromised patient (2), and bacteremia from contamination of a central venous catheter (3). Analysis of a collection of 87 clinical isolates showed that $62 \%$ had been recovered from respiratory tracts (without clinical significance); other isolates had been responsible for catheter sepsis, pneumonia, subcutaneous abscess, cellulitis, osteomyelitis, lymphadenitis, surgical wound infection, and peritonitis (4).

In conclusion, we report a culture-negative peritonitis caused by M. mucogenicum, which responded to combination therapy within 10 days. The infection was diagnosed with the help of polymerase chain reaction and culture techniques, permitting prompt and appropriate therapy to be instituted, preventing excessive morbidity and preserving peritoneal membrane function.

\section{DISCLOSURES}

The authors have no financial conflicts of interest to declare. 


$$
\begin{array}{r}
\text { Madras Medical Mission Hospital }{ }^{1} \\
\text { Chennai, India }^{2} \\
\text { Pondicherry Institute of Medical Sciences }{ }^{2} \\
\text { Pondicherry, India } \\
\text { *email:abraham_georgi@yahoo.com }
\end{array}
$$

\section{REFERENCES}

1. Springer B, Böttger EC, Kirschner P, Wallace RJ Jr. Phylogeny of the Mycobacterium chelonae-like organism based on partial sequencing of the 16S rRNA gene and proposal of Mycobacterium mucogenicum sp. nov. Int J Syst Bacteriol $1995 ; 45: 262-7$.

2. Shehan JM, Sarma DP. Mycobacterium mucogenicum: report of a skin infection associated with etanercept. Dermatol Online J 2008; 14:5.

3. Kline S, Cameron S, Streifel A, Yakrus MA, Kairis F, Peacock $\mathrm{K}$, et al. An outbreak of bacteremias associated with Mycobacterium mucogenicum in a hospital water supply. Infect Control Hosp Epidemiol 2004; 25:1042-9.

4. Wallace RJ Jr, Brown BA, Griffith DE. Nosocomial outbreaks/pseudo-outbreaks caused by nontuberculous mycobacteria. Annu Rev Microbiol 1998; 52:453-90. 